\title{
EDITIONS
}

\section{DU CENTRE NATIONAL DE LA RECHERCHE SCIENTIFIQUE}

\section{I. - PUBLICATIONS PERIODIQUES}

\section{IE BULLETIN SIGNALÉTIQUE}

$3^{\text {e }}$ Partie (trimestrielle) consacrée à la Purlosoprrie et aux

Sciences humaines : Abonnements. - France......... 4000 F Etranger......... $5000 \mathrm{~F}$

Abonnement au "Centre de Documentation du C.N.R.S."

16, rue Pierre-Curie, Paris-Ve, tél. DANton 87-20; C.C.P. Paris 9131-62

\section{BIBLIOGRAPHIE ANNUELLE DE L'HISTOIRE DE FRANCE}

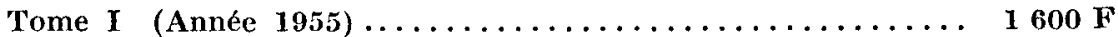

Tome II (Année 1956) ....................... $2200 \mathrm{~F}$

ARCHIVES DE SOCIOLOGIE DES RELIGIONS. Cette revue semestrielle est publiée par le Groupe de Sociologie des Religions.

Le $n^{\circ}$ de 192 à 224 pages.................... $1000 \mathrm{~F}$

Abonnement annuel $\left(2 n^{\mathrm{os}}\right) \ldots \ldots \ldots \ldots \ldots \ldots \ldots \ldots \ldots \ldots \ldots \ldots \ldots$

$$
\text { II. - OUVRAGES }
$$

\section{ÉTUDES SOCIOLOGIQUES}

Les travailleurs algériens en France (relié pleine toile grise). $1200 \mathrm{~F}$

\section{ÉTUDES HISTORIQUES}

G. Lefebvre et A. 'Terroine, Recueil des Documents relatis aux séances des États Généraux de $1789 \ldots \ldots \ldots \ldots 2500 \mathrm{~F}$

P. Mantoux, Les délibérations du Conseil des Quatre (24 mars-28 juin 1919).

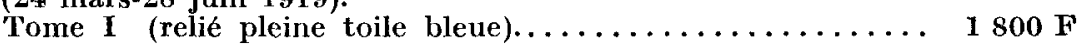

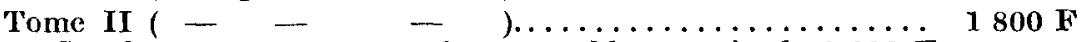
Ces deux tomes sont vendus ensemble au prix de $3200 \mathrm{~F}$

\section{Collection LE GHCEUR DES MUSES}

2. La IMsique Instrumentale de la Renaissance (relié pleine toile crème), in-4, $394 \mathrm{p} \ldots \ldots \ldots \ldots \ldots \ldots \ldots \ldots, 1800 \mathrm{~F}$

3. Les Fêtes de la Renaissance (relié pleine toile crème),

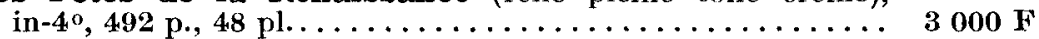

4. La Renaissance dans les Provinces du Nord (relié pleine toile crème), in-4., 219 p..................... $1100 \mathrm{~F}$

\section{III. - COLLOQUES INTERNATIONAUX}

V. - Musique et Poésie au XVIe siècle............. I $600 \mathrm{~F}$

VII. - Sociologie Comparée de la Famille contemporaine. $1000 \mathrm{~F}$

Renseignements et Vente : Service des Publications du C.N.R.S., 13, quai Anatole-France, Paris-VIIe, tél. INV. 45-95, C.C.P. Paris 9061-11. 


\section{LE XIe CONGRÈS INTERNATIONAL DES SCIENCES HISTORIQUES}

\section{STOCKHOLM， 1960}

L'information que nous avons publiée dans un précédent numéro à propos de ce Congrès, n'était pas complète. Le Secrétariat du Comité français des Sciences historiques nous fournit la précision suivante : les ORGANISMES INTERNATIONAUX AFFILIES AU COMITÉ INTERNATIONAL DES SCIENCES HISTORIQUES :

Association internationale des Etudes byzantines ;

Commission internationale des Études slaves ;

Instituto panamericano de Geografia e Historia ;

Comité international des Sciences onomastiques,

et les COMMISSIONS DU COMITÉ INTERNATIONAL DES SCIENCES HISTORIQUES :

Commission de Bibliographie internationale;

Commission d'Histoire diplomatique;

Commission internationale de Numismatique;

Commission internationale pour l'Histoire des Assemblées d'État ;

Commission d'Histoire militaire comparće ;

Commission internationale d'Histoire ecclésiastique ;

Commission d'Histoire des mouvements sociaux ;

Commission de Démographie historique ;

Commission d'Iconographie;

Commission des Archives du Vatican,

participant au Congrès, organiseront, chacune pour leur compte, des séances de travail, dont le programme n'est pas encore arrêté et sera ultérieurement communiqué.

Directeur de la publication : A. ELISSABIDE Imprimerie Tournon et Cie, 20, rue Delambre, Paris (14.5) - 1० trimestre 1958. - I. N० 925. 


\section{Collection " L'Evolution de l'Humanité "}

\section{LUCIEN FEBVRE ET H.-J. MARTIN}

\section{L'APPARITION DU LIVRE}

«Etablir comment et pourquoi le livre imprimé a été tout autre chose qu'une réalisation technique commode et d'une ingénieuse simplicité : la mise au point d'un des instruments les plus puissants dont ait pu disposer la Civilisation d'Occident pour concentrer la pensée éparse de ses représentants: donner toute son efficacité à la méditation individuelle des chercheurs en la transmettant aussitôt à d'autres chercheurs; réunir à la convenance de chacun, et sans délai, ni peine, ni frais, ce concile permanent de grands esprits dont a parlé Michelet, en termes impérissables; lui procurer ainsi une vigueur centuplée, une cohérence toute nouvelle, et par là même une puissance incomparable de rayonnement... bref, montrer dans le Livre l'un des moyens les plus efficaces de cette maîtrise sur le monde, tel est le but de cet ouvrage. »

Extrait de la Préface de LUCIEN Febvre.

I vol. in $-8^{\circ}, 560$ pages, $2.400 \mathrm{~F} .+\mathrm{T} . \mathrm{L}$. 


\section{BIBLIOTHEQUE GENERALE DE LECOLE PRATIQUL' \\ DES II.IUTES ETUDES (IT SECTION)}

Sriences Ficonomiques et Sociales

\section{LÉOPOLD CHATENAY}

\section{VIE DE JACQUES ESPRINCHARD, ROCHELAIS, ET JOURNAI, DE SES VOYAGES AU XVI ${ }^{\mathrm{e}}$ SIECLE}

Jacques Esprinchard, sieur du Plom, gentilhomme rochelais, au demeurant lıonnête écrivain provincial qui a publié des traductions, des histoires (Empereurs romains, ottomans), a entrepris en 1597-1598 un grand voyage à travers l'Europe : Leyde, Arnheim, Cologne, Francfort, Nuremberg, Magdebourg, I eipzig, Cracovic, Vienne, Prague, Ratisbonne, Munich, Ulm, Heidelberg, Strasbourg, Bâle, Genc̀ve. Il a parcouru aussi la France méridionale, de Lyon i. Valence, Avignon, Montpellier, Carcassonne, Toulouse, Pau, Dax, Bordeaux, promenant partout une curiosité inlassable, gentiment érudite, qui évoque assez bien cet autre voyageur, qui fut presque son contemporain et presque son compatriote : Montaigne.

Un volume de 308 pages $\ldots \ldots \ldots \ldots \ldots \ldots \ldots \ldots \ldots \ldots \ldots \ldots \ldots 1800 \mathbf{F}$ liranco.................................. 1940 F

En rente an S.E.V.P.F.N., 13, rue du Four, PARIS-VIe

\section{PUBLICATIONS}

DU CEN'TRE DE RECHERCHES HISTORIQUES

COLIECTION"AFFAIRES ET GENS D'AFFAIRES "

$$
-12-
$$

\section{BERNARD SCHNAPPER}

\section{L.ES RENTES AU XVI SIECLE \\ Histoire d'un instrument de crédit}

A une époque où le prêt à intérêt est interdit, les rentes jouent daus l'économie un rôle dont l'importance $n^{*}$ avait pas encore été mesurée. Elles sont aussi au centre des préoccupations des canonistes. ('e livre étudie done une transformation juridique et leur application pratique au sein d'une économie en plein renouveau.

In volume de $3 \mathrm{I} 2$ pages.................. $1500 \mathrm{~F}$

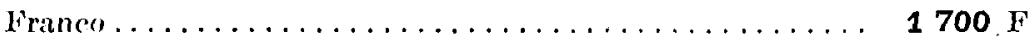

S.E.V.P.E.N. 


\section{MARC BLOCH}

\section{L'étrange défaite}

TÉmoignage ÉCrit EN 1940

suivi de

\section{ECrits clandestins (1942-1944)}

"Il est admirable qu'un grand témoin, tombé quatre ans après au service de la Résistance, ait pu découvrir, analyser avec cette clarté les secrets de la plus étrange des défaites. Nous n'hésitons pas à dire qu'il n'a pas paru à ce jour en France, sur 1940, un récit, une explication, un réquisitoire d'une pensée aussi lucide, d'un dessin aussi net."

Georges ALTMANN, dans I'Avant-Propos.

Les Ecrits Clandestins sont des textes inédits, extraits des Cahiers politiques, organe clandestin du Comité Général d'Etudes de la Résistance :

- Pourquoi je suis un républicain, juillet 1943.

- A propos d'un livre trop peu connu (Général Chauvineau : Une invasion est-elle encore possible?), avril 1944.

- La vraie saison des Juges, 1944.

- Sur la réforme de l'enseignement, 1944.

Editions Albin Michel

22, rue Huyghens, 22

PARIS 


\section{CENTRE D'ÉTUDES DES AIRES CULTURELLES}

\section{COLIECTION « LE MONDE D'OUTRE-MER PASSE ET PRESENT »}

\section{I. - ÉTUDES}

1. Louis DUMONT, Une sous-caste de l'Inde du Sud. Organisation sociale et religion des Pramalai Kallar..............4 $290 \mathrm{~F}$

2. Jacques BERQUE, Al-Yousi, Problèmes de la culture maro-

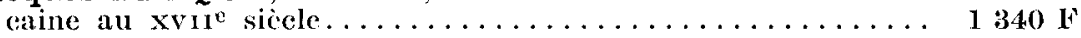

3. Jacques BERQUE, Histoire sociale d'un village égyptien au

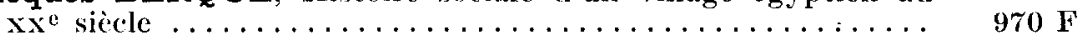

Sous presse :

4. TUNGTSU GHU, Law and society in Traditional china.

5. Roger BASTIDE, Le candomblé de Bahia (rite nagô).

6. E. NARACHI, Recherches démographiques dans les pays sous-développés (notamment en Iran).

7. G. VREEDE-DE STUERS, L'émancipation de la femme indonésienne.

\section{II. - DOGUIMENTS}

Sous presse :

1. A. GREIS, La révolution d'Oraby Pacha.

2. VOLNEY, Voyage en Egypte et en Syrie. Publié avec une Introduction et des notes par Jean Gaulmier.

$$
\text { III. - ESSAIS }
$$

\section{Sous presse:}

1. H. DE MONTETY, Femmes de Tunisie.

2. T. TOUMAa, Un village de montagne au Liban : Hadeth el-Jobbé.

$$
\text { IV. - BIBLIOGRAPHIES }
$$

1. Y. HERVOUET, Catalogue des monographies locales chinoises dans les bibliothèques d'Europe................. 2150 F

Sous presse :

2. Y. HERVOUET, Cataloguc des périodiques chinois dans les bibliothèques d'Europe.

3. An Anthropological Bibliography of South-East Asia, together with a Directory of Recent Anthropological Field Research. Compiled by Elizabeth von Fürer-Haimendorf. With a Foreword by Christoph von liürer-Haimendorf.

\section{IEDITIONS MOUTON ET Cie \\ PARIS - LA HAYE}




\section{COLLECTION « SOCIOLOGIE ET ÉCONOMIE DES PAYS SLAVES »}

1. G. BOBROWSKI, Formation du système soviétique de plani-

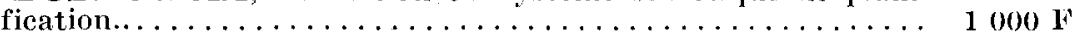

Sous presse:

2. ROBERT E. F. SMITH, Farming in Russia.

\section{COLLECTION « DOCUMENTS ET RECHERCHES SUR L'ÉCONOMIE DES PAYS BYZANTINS, ISLAMIQUES E'T SLAVES, AU MOYEN AGE »}

Sous presse :

1. F. THIRIET; Régestes des délibérations du Sénat de Venise concernant la Romanie, t. 1: 1329-1399.

\section{REVUES}

Contributions to Indian Sociology, edited by Louis Dumont (Paris) and D. Pocock (Oxford), no $1 \ldots \ldots \ldots \ldots \ldots \ldots \ldots$ roo F

Revue Bibliographique de Sinologie, $n^{0} 1,1955 \ldots \ldots \ldots \ldots \ldots 4000 \mathrm{~F}$

\section{LOUIS DUMONT}

\section{UNE SOUS-CASTE DE L'INDE DU SUD}

\section{Organisation sociale et Religion des Pramalai Kallar}

La présente étude est fondée sur un postulat qu'elle vérifie : les castes ne sont pas des systèmes sociaux différents participant de façon sociologiquement insaisissable à une civilisation, à une religion, à un langage communs. Toutes les castes d'une aire culturelle donnée - ici l'aire linguistique tamoule - reposent sur des institutions fondamentales communes, qu'il s'agit de découvrir sous les diversités individuelles, et qui constituent, avec le systìme des castes lui-même, la morphologie sociale de la civilisation considérée. Extrait de l'introduction de LOUIS DUMONT.

Un volume de $\mathrm{xrv-460} \mathrm{p} ., 38 \mathrm{pl}$. $4.285 \mathrm{~F}$

\section{MOUTON et Cic \\ Paris-La IIaye}

En vente :

IIBRAIRIE MAISONNEUVI

198, boulevard Saint.Germain

PARIS-VI ${ }^{\circ}$ 
TABLEAUX STATISTIQUES RELATIFS A L'ECONOMIE FRANÇAISE ET L'ÉCONOMIE MONDIALE

Col ouvrage est le complément de

Forces et Faiblesses de l'économie française par J.-M. JEANNENEY . . . . . . I 000 F

\section{INVESTISSEMENT ET FINANCEMENT}

Origine et cmploi des jonds de grandes sociétés par M. MALISSEN

\section{la Chambre des lords AU XX・ SIECLE}

L'étude originale de cet auteur nous permet

de voir viare cette institution

par M. BOUISSOU. .

$1300 \mathrm{~F}$

\section{TEXTES DE DROIT ECONOMIQUE}

\section{ET SOCIAL FRANÇAIS}

De la nuit du + toût au Marché commun par J.-M. JEANNENEY et M. PERROT cartonné $2900 \mathrm{~F}$

\section{LA COLLCTIVISATION DE L'AGRICULTURE}

Histoire de la collectivisation de Lénine à

Mao T'sé Tung, en U. R. S. S., dans les pays

du Kuminjorm, en Yougoslavie et en Chine par Ch. BOUVIER. 


\title{
DIOGENE
}

Revee Internationale des SCIEnche Humanes

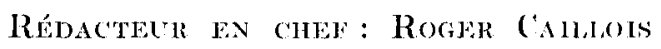

\author{
No.2. ; AVRIL_JUIN 19:5
}

Sommitrs:

JaN De Vrass.......... Les Comles populates

Creme M. Bowna......... Pósie et:Traditions

Georges-Devent....... Les Mobiles humeins de linistoire

Pierre Auglir .......... Le Régime des Castes doms les Populations d'Idécs

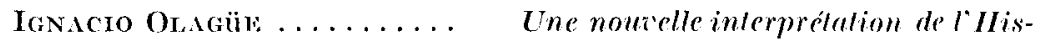
loire

Chroniques

Andrú Dupont-Sommer ... Problemes des Mamuscrits de la mer Morte

Marc Chapiro........... Angoisse ef Sociéte

\section{REDACTION E'T ADMINISTRATION}

19, avenue Kréber, Paris-16"

(Kléber 52-00)

Revee trimestrielle paraissant en six-langues : allemend, anglais, arabe, espagnol, français_et_italien.

L'Édition française est publiée- par la Librairic Gallimard, :5, me Sébastien-Bottin, Paris-re.

Les abonnements sont souserits auprès de cette maison ('.e.p. 169.33, Paris).

Prix de vente au numéro : 240 francs.

Tarifs d'abonnement : France $840 \mathrm{fr}$. ; étranger 1.050 $\mathrm{fr}$. 


\title{
GEORGES CONDOMINAS
}

\section{NOUS AVONS MANGÉ LA FORÊT}

\author{
Chronique de Sar Luk, village Mnong Gar \\ des Hauts-Plateaux du Viet-Nam Central
}

Nous 'avons mangé la Forêt de la Pierre-Génie Gôo est la traduction littérale de l'expression mnong gar Hii saa Brii Mau - Yaang Gôo, qui désigne à Sar Luk l'année agricole qui va de fin novembre 1948 à début décembre 1949. Les Mnong Gar sont en effet des écobuants semi-nomades des Hauts-Plateaux vietnamiens, qui désignent chaque année du nom de la forêt qu'ils ont « mangée ", c'est-à-dire abattue et incendiée pour y faire leurs cultures annuelles.

Ce livre est donc la simple chronique du village de Sar Luk; il décrit par le détail un certain nombre de journées d'un village mnong gar, prises dans le déroulement du cycle agricole annuel. On assiste ainsi à la consécration d'une alliance par un double sacrifice du buffle, au « jugement » d'un inceste suivi d'un suicide, à des séances de chamanisme, un mariage, une naissance, un décès (avec les rites funéraires et le partage de l'héritage auquel il a donné lieu), etc., avec en toile de fond les différentes étapes de la vie agricole avec ses techniques et ses rites, notamment la mise à feu de quarante hectares de forêt, une grandiose Fête du Sol au cours de laquelle onze huffles sont immolés, la moisson et la recherche d'une nouvelle forêt à " manger $» . .$.

Un volume de 528 pages, 45 photographies de l'auteur, cartes, dessins, plans .................... $1500 \mathrm{~F}$

\section{LE MERGURE DE FRANCE}

26, rue de Condé

PARIS-VIP 


\section{ANNALES \\ ÉCONOMIES - SOCIÉTÉS - CIVILISATIONS}

Rédaction et Administration: LIBRAIRIE ARMAND COLIN, 103, Bd St-Michel, PARIS-V• Compte de chèques nostaux : Paris, No 1671

Abonnements 1958 : France et Union française, $1700 \mathrm{~F}$; Etranger, $2000 \mathrm{~F}$

Le numéro de 208 pages : $450 \mathrm{~F}$

Années parues : Année 1957, . . . . . 1500 F. - Chaque numéro, . . . . . . . 400 F Chaque année antérieure, $1200 \mathrm{~F}$. C Chaque numéro, . . . . . . $325 \mathrm{~F}$

ANNALES D'HISTOIRE ÉCONOMIQUE ET SOCIALE (1929-1938)

Chaque Année parue....... 1.200 F - Le numéro des Anuées parues....... 325 F ANNALES D'HISTOIRE SOCIALE (1939-1941). Chaque Année.......... 1200 F MÉLANGES D'HISTOIRE SOCIALE (1942-1945), Chaque Année......... 1200 F Table analytique des Annales $1929-1949$ avec un supplément 1950-1951..... 1200 F

\section{CAHIERS DES ANNALES}

1. P. Sardilia, Nouvelles et spéculations à Venise au début du XVIe siècle ...........................

2. Ch. Morazí, Trois essais sur Histoire et Culture .......

3. Marc Bloch, Apologie pour l'Histoire ou Métier d'Historien,

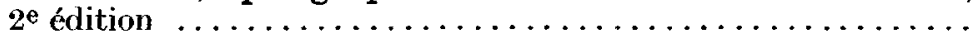

4. A travers les Amériques latines (Recucil publié sous la direction de Lucien Febvre) .................. 625 F

5. Georges Friedmann, Humanisme du travail et humanités. Epuisé.

6. L. E. HalKIN, Initiation à la critique historique, $2^{e}$ édition $600 \mathrm{~F}$

7. G. Debien, En haut Poitou : défricheurs au travail (XVe.

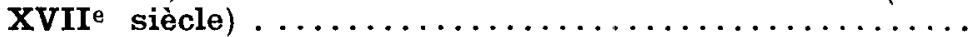

8. A. Tenenti, La Vie et la Mort à travers l'art du XVe siècle

9. Marc Bloch, Esquisse d'une histoire monétaire de l'Europe $375 \mathrm{~F}$ $530 \mathrm{~F}$ $325 \mathrm{~F}$

10. A. Koyné, Mystiques, Spirituels, Alchimistes du XVIe siècle allemand $\ldots \ldots \ldots \ldots \ldots \ldots \ldots \ldots \ldots \ldots \ldots \ldots \ldots \ldots \ldots$

11. G. Debien, Etudes antillaises au XVIIIe siècle $\ldots \ldots \ldots$ r00 F Sous presse :

12. F. L'Huillier, La lutte ouvrière à la fin du Second Empire. A paraitre :

13. Makc Bloci, La France sous les derniers Gapétiens (1223-1328).

\section{REVUE ÉCONOMIQUE \\ Publiêe avec le concours de la VIe Section de l'École pratique des Hautes Études et du C. N. R.S.}

Comité de direction : † A. Aftalion, R. Barre, F. Braudel, P. Coutin, E. James, E. Labrousse, J. Lhomme, A. Marchal, J. Marchal, J. Meynaud, H. Noyelle, J. Weiller

Abonnement : France et Union Française, 2500 F Etranger, $2900 \mathrm{~F}$ 1958

Le numéro..... $600 \mathrm{~F}$

L I B R A I R I A R A N D C OL I N 


\section{COLLECTION DIRIGÉE}

\section{PAR \\ Lucien FEBVRE \\ Membre de l'Institut

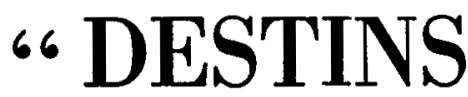 \\ Fernand BRaUdeL \\ Professeur au Collège de France

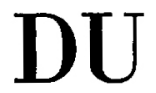 \\ "Il ne s'agit pas de refaire cette Histoire Universelle de Lavisse et Rambaud qui, en d'autres temps, dans un autre climat, rendit tant de services aux historiens... \\ ... Mieux vaut réduire notre ambi- tion à poser en termes corrects, un certain nombre de grands problemes qui nous inquiètent et nous hantent." \\ Lucien FEBVRE (Annales, 1954).}

Premier volume paru :
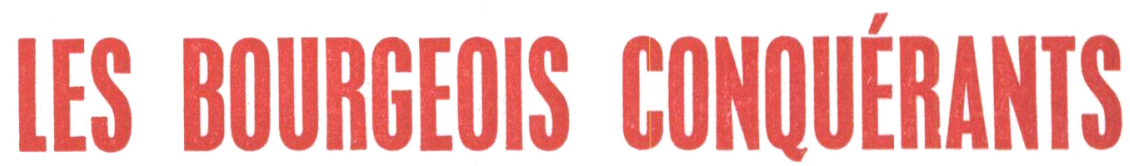

\section{par Charles MORAZE}

"Si vous voulez, votre manuscrit joint les qualitês d'un morceau d'histoire d'une singulière richesse aux vertus d'un essai brillant et séduisant. $J$ 'en suis ravi.

Je dirai que, pour la premiere fois, $j$ 'ai lu un livre d'histoire b́conomique et sociale conforme dे ce que ce nom doit désigner.

\section{Lucien FEBVRE}

Un volume in-4.0, 506 pages, 40 héliogravures, dont 8 en coulours, 38 cartes et plans, 3 index de personnes, de lieux, de choses, 1 tableau chronologique.

Relié pleine toile sous jaquette en couleurs.......... $4900 \mathrm{~F}$ Exemplaire broché....................... 4500 F

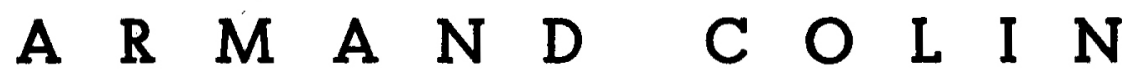

